\title{
Metallodielectric Photonic Crystal Resonators
}

\author{
Ali Serpengüzel* \\ Koç University, Microphotonics Research Laboratory, Physics Department, Rumeli Feneri Yolu, \\ Sariyer, Istanbul 34450 Turkey
}

\begin{abstract}
A resonator has been manufactured using a photonic crystal at $\mathrm{K}_{\mathrm{u}}$ band frequencies. The photonic crystal has a face centered cubic Bravais lattice structure. In the transmission measurements, the photonic crystal displayed a directional bandgap with a lower band edge of $13.0 \mathrm{GHz}$, an upper band edge of $21.5 \mathrm{GHz}$, and a center frequency of $17.25 \mathrm{GHz}$. The corresponding stop bandwidth center frequency ratio is $50 \%$. The maximum rejection at the band center is $35 \mathrm{~dB}$. The unit cell rejection ratio is $7 \mathrm{~dB}$ per unit cell. The resonance has a quality factor of 200 , and a maximum transmission peak of $-5 \mathrm{~dB}$.
\end{abstract}

Keywords: photonic crystals, resonators.

\section{INTRODUCTION}

Photonic crystals are attracting the attention of the photonics community. ${ }^{1-2}$ They are constructed by spatially patterning the permittivity of a medium in a periodic way. Such a medium exhibits photonic stop bands for certain electromagnetic frequencies. ${ }^{3}$ Photons with these frequencies cannot propagate in the medium, thereby enabling the control of the transmission characteristics of the medium. Three-dimensional semiconductor photonic crystals have been recently realized in the optical and near-infrared. ${ }^{4}$ Metallodielectric photonic crystals have also been fabricated in the far-IR regime ${ }^{5}$. In addition to the visible and IR, photonic crystals have also found applications in the microwave and millimeter wave regime such as high directivity antennas ${ }^{6-7}$ high quality resonators ${ }^{8}$, accelerator cavities ${ }^{9}$, as well as high power ${ }^{10}$ and broadband ${ }^{11}$ reflectors. Dielectric photonic crystals composed of spherical ${ }^{12}$ and non-spherical 13,14 atoms have been investigated in the microwave regime. The stop bandwidth of these photonic crystals can be enhanced using metals, which have large imaginary refractive indices at microwave and millimeter frequencies. Metallodielectric photonic crystals composed of flat atom metal elements ${ }^{15}$, metallic island elements ${ }^{16}$, wire meshes ${ }^{17}$, and spheres have already been investigated experimentally ${ }^{18}$ and theoretically ${ }^{19}$ at microwave frequencies. However, some applications require the inclusion of defects in the metallic photonic crystal to select a transmitting state within the stop band ${ }^{20}$. These defects can be zero-dimensional for cavity ${ }^{21}$, one-dimensional for waveguide ${ }^{22}$, and twodimensional for resonator applications. The metallodielectric photonic crystal with the resonator geometry can be applied to microwave tubes, such as klystrons, magnetrons, cross-field amplifiers, and traveling wave tubes. ${ }^{23}$ Additional resonator applications may include microwave spectrum analyzers, or MASER cavities.

\section{EXPERIMENTAL SETUP}

In the experiments, we have investigated a 3D metallodielectric photonic crystal in the microwave regime. ${ }^{24}$ The metallodielectric photonic crystal has an fcc Bravais lattice composed of steel spheres, with diameter $\mathbf{d}=6.35 \mathrm{~mm}$. The lattice was formed along the (100) direction by stacking alternating layers of steel spheres. One layer consists of spheres packed as shown in Fig.1 (a), which depicts the (001) plane (top view) of the metallodielectric photonic crystal. The layer above and below that layer has the same layout, but they are offset by half a lattice constant in both horizontal directions. Our metallodielectric photonic crystal consisted of 11 layers or 5 unit cells. The lattice constant was $\mathbf{a}=15$ $\mathrm{mm}$, corresponding to a packing fraction of $\mathbf{f}=0.16$. The supporting dielectric material was air doped polystyrene with a refractive index of 1.16 .

* aserpenguzel@ku.edu.tr; phone: + 90 (212) 338-1312; fax: + 90 (212) 338-1547, http://home.ku.edu.tr/ aserpenguzel, http://microphotonics.ku.edu.tr, Koç University, Microphotonics Research Laboratory, Physics Department, Rumeli Feneri Yolu, Sariyer, Istanbul 34450 Turkey. 


\section{THE MICROWAVE PHOTONIC CRYSTAL}

The transmission through the metallodielectric photonic crystal was measured from 9.5 to $24.5 \mathrm{GHz}$ with a network analyzer. Standard gain horn antennas were used to transmit and receive EM radiation. Surroundings of the test setup was covered with absorbers to build an anechoic chamber resulting in a sensitivity of $85 \mathrm{~dB}$. The transmission calibration was performed using the standard gain horn antennas, before placing the photonic crystal in the microwave beam. We first measured the transmission properties of the metallodielectric photonic crystal without the resonator. Figure 1(b) shows the transmission spectrum through the metallodielectric photonic crystal along the (100) crystal direction. The lower edge of the stop band starts at $13.0 \mathrm{GHz}$. The center frequency for the stop band is at $17.25 \mathrm{GHz}$, which compares favorably with the normal $\left(\boldsymbol{\theta}=0^{\circ}\right)$ incidence Bragg condition frequency $\nu_{\mathrm{Bragg}}=\mathbf{c} / \mathbf{2} \operatorname{ancos}(\boldsymbol{\theta})$, where $\mathbf{c}$ is the speed of light in vacuum, $\mathbf{n}$ is the refractive index of the metallodielectric medium, and $\boldsymbol{\theta}$ is the angle between the (100) crystal direction and the k-vector of the incident EM wave. The upper edge of the stopband is at $21.5 \mathrm{GHz}$. Therefore, the width of the stopband is $50 \%$ of the center frequency, which makes our metallodielectric photonic crystal suitable for microwave applications. Additionally, the upper edge and the lower edge of the stopband compare favorably to the frequencies estimated using the calculations of Ref 19. Ignoring the oscillations, the maximum rejection at the band center is $35 \mathrm{~dB}$, corresponding to a rejection of $7 \mathrm{~dB}$ per unit cell. The transmission below and above the stop band is unity, which is remarkable considering that a metallic surface would have reflected $99.97 \%$ of the incident radiation in the given microwave frequency range.
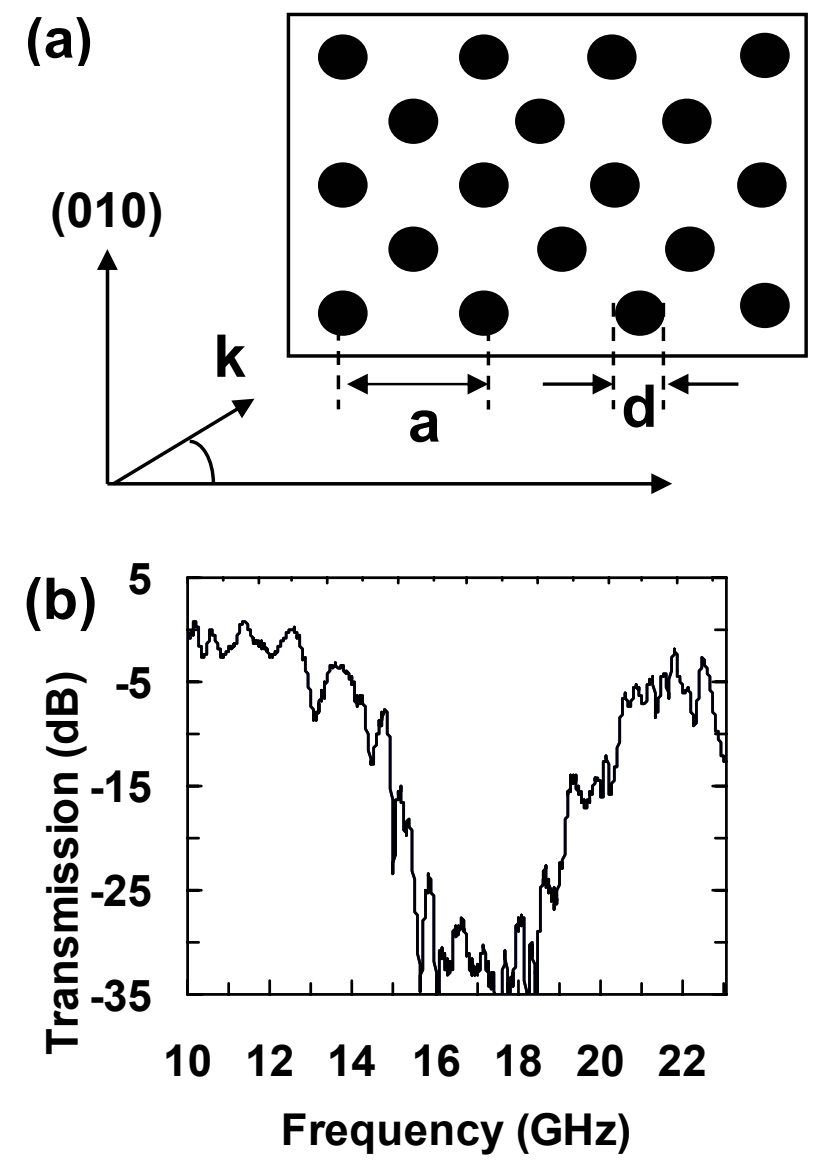

Fig. 1. (a) The top view of the (001) oriented face of the metallodielectric photonic crystal, (b) normal $0^{\circ}$ (100) incidence transmission through the metallodielectric photonic crystal. 


\section{THE MICROWAVE RESONATOR}

We later measured the transmission properties of the fcc metallodielectric photonic crystal with the resonator. The resonator is formed by separating the photonic crystal into two separate identical blocks each consisting of 5 layers, where each 5 layer stack acts like the mirror of a resonator. The separation distance is set to be $\mathbf{L}=17.5 \mathrm{~mm}$ between the 2 parts (mirrors) of the metallodielectric photonic crystal. Figure 3 (a) shows the transmission spectrum of the metallodielectric photonic crystal at low spectral resolution and along (100) crystal direction. The spectrum in Fig. 2 (a) is very similar to the full metallodielectric photonic crystal spectrum of Fig. 1 (b), except the mode at frequency $\mathbf{v}=$ $16.02 \mathrm{GHz}$. Fig. 2 (b) shows the transmission spectrum of the metallodielectric photonic crystal at high spectral resolution for the (100) crystal direction. The mode at frequency $\mathbf{v}=16.02 \mathrm{GHz}$, has a linewidth of $\Delta \mathbf{v}=0.08 \mathrm{GHz}$, and a quality factor of $\mathbf{Q}=\mathbf{v} / \boldsymbol{\Delta v}=200$. The mode frequency of $16.02 \mathrm{GHz}$ compares favorably with the resonance condition of $\mathbf{v}=\mathbf{c} \mathbf{\Pi m}-\boldsymbol{\varphi} / \mathbf{4} \Pi \mathbf{L}$, where $\mathbf{m}$ is the mode number, $\boldsymbol{\varphi}$ is the total reflection phase of the two mirrors, and $\mathbf{L}$ is the separation distance ${ }^{25}$. From the frequency of the resonance the mode number is found to be $\mathbf{m}=2$ and the total reflection phase of the two mirrors $\varphi=47^{\circ}$.
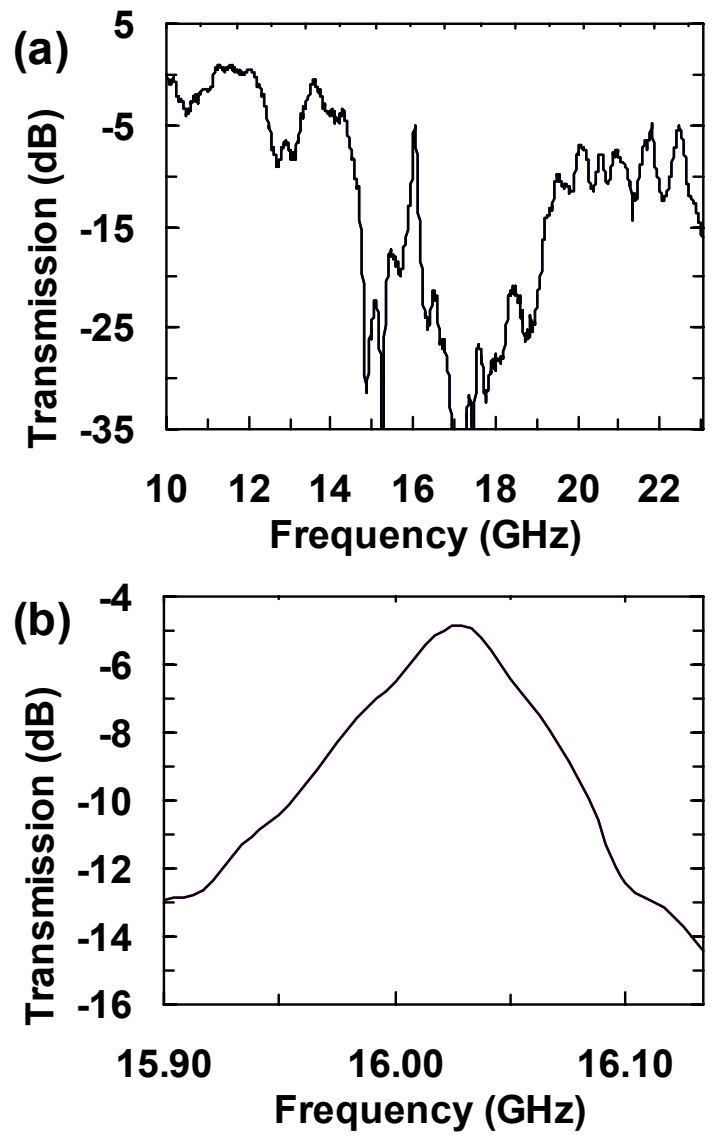

Fig. 2. (a) normal $0^{\circ}(100)$ incidence transmission through the metallodielectric fcc photonic crystal with the gap in the middle, (b) expanded frequency scale of the spectrum of fig. 2 (a).

\section{CONCLUSIONS}

In conclusion, a resonance has been observed in the stop band of a metallodielectric photonic crystal by transmission measurements at microwave frequencies. By choosing the propagation direction perpendicular to the gap, and with the appropriate tuning mechanism, the resonator geometry can be used as a microwave spectrum analyzer or, alternatively, 
a MASER cavity. However, by choosing the propagation direction parallel to the gap, we obtain the waveguide geometry ${ }^{26}$, which can be used to guide the microwave in the photonic crystal. The inclusion of a gap defect in the metallodielectric photonic crystal provides further physical insight on the behavior of the electromagnetic modes of this type of photonic crystal. More realistic applications for the metallodielectric photonic crystal can be envisioned in microwave tubes, such as magnetrons, klystrons, cross-field amplifiers, and traveling wave tubes. These microwave tube devices are still in use today, mostly in satellite communication and RADAR's.

The periodic coupled cavity structure already present in the traveling wave tubes can be improved by the incorporation of the metallodielectric photonic crystal. The resonator geometry may provide additional RF selectivity for the input and output cavity of the klystron. The incorporation of the metallodielectric photonic crystal in the anode of the magnetron, or the slow wave structure of the cross-field amplifiers could increase the frequency selectivity of these microwave devices. Additionally, the fabrication of these microwave devices using the metallodielectric photonic crystal may improve the packaging weight, size, thermal management, and lower cost.

\section{ACKNOWLEDGMENTS}

The author would like to acknowledge the partial support of this work by the NATO Grant No: SfP-971970 and the British Council Academic Link Grant.

\section{REFERENCES}

1 E. Yablonovitch, Phys. Rev. Lett. 58, 2059 (1987).

2 S. John, Rev. Lett. 58, 2486 (1987).

3 J. D. Joannopoulos, R. D. Meade, and J. N. Winn, Photonic Crystals, (Princeton University Press, New Jersey, USA, 1995). pp. 45-104.

$4 \quad$ S.Y. Lin and J. G. Fleming, J. Lightwave Technol. 17, 1944 (1999).

5 K. A. McIntosh, L. J. Mahoney, K. M. Movar, O. B. McMahon, S. Verghese, M. Rothschild, and E. R. Brown, Appl. Phys. Lett. 70, 2937 (1997).

6 S. D. Cheng, R. Biswas, E. Özbay, J. S. McCalmont, G. Tuttle, and K. M. Ho, Appl. Phys. Lett. 64, 3399 (1995).

7 E. R. Brown and O. B. McMahon, Appl. Phys. Lett. 68, 1300 (1996).

8 C. J. Maggiore, A. M. Clogston, G. Spalek, W. C. Sailor, and F. M. Mueller, Appl. Phys. Lett. 65, 1451 (1994).

9 D. R. Smith, S. Shultz, N. Kroll, M. Sigalas, K. M. Ho, and C. M. Soukoulis, Appl. Phys. Lett. 65, 645 (1994).

10 K. Agi, L.D. Moreland, E. Schamiloglu, M. Mojahedi, K. J. Malloy, and E. R. Brown, IEEE Trans. Plasma Sci. 24, 1067 (1996).

11 K. Agi, E. R. Brown, O. B. McMahon, C. Dill III, and K. J. Malloy, Electron. Lett. 30, 2166 (1994).

12 E. Yablonovitch and T. J. Gmitter, J. Opt. Soc. Am. A 7, 1792 (1990).

13 E. Yablonovitch, T. J. Gmitter, and K. M. Leung, Phys. Rev. Lett. 67, 2295 (1991).

14 E. Özbay, E. Michel, G. Tuttle, M. Sigalas, R. Biswas, and K. M. Ho, Appl. Phys. Lett. 64, 2059 (1994).

15 A. Kao, K.A. McIntosh, O. B. McMahon, R. Atkins, and S. Verghese, Appl. Phys. Lett. 73, 145 (1998).

16 D. F. Sievenpiper, E. Yablonovitch, J. N. Winn, S. Fan, P. R. Villeneuve, and J. D. Joannopoulos, Phys. Rev. Lett. 80, 2829 (1998).

17 D. F. Sievenpiper, M. E. Sickmiller, and E. Yablonovitch, Phys. Rev. Lett. 76, 2480 (1996).

18 E. R. Brown and O. B. McMahon, Appl. Phys. Lett. 67, 2138 (1995).

19 S. H. Fan, P. R. Villeneuve, J. D. Joannopoulos, Phys. Rev. B 54, 11245 (1996).

20 M. M. Sigalas, C. T. Chan, K. M. Ho, and C. M. Soukoulis, Phys. Rev. B 52, 11744 (1995).

21 W. Y. Leung, G. Tuttle, M. M. Sigalas, R. Biswas, K. M. Ho, and C. M. Soukoulis, J. Appl. Phys. 84, 4091 (1998).

22 M. M. Sigalas, R. Biswas, K. M. Ho, and C. M. Soukoulis, Phys. Rev. B 60, 4426 (1999).

23 L. Sivan, Microwave Tube Transmitters, (Chapman and Hall, London, UK, 1994). pp. 37-221.

24 A. Serpengüzel, IEEE Microwave Wireless Components Lett. 12, 134-136 (2002).

25 E. Özbay and B. Temelkuran, Appl. Phys. Lett. 69, 743 (1996).

26 J. D. Joannopoulos, P. R. Villeneuve, and S. H. Fan, Nature 386, 143 (1997). 